\title{
Robotic Path Planning and Visibility with Limited Sensor Data
}

\author{
Yanina Landa ${ }^{1}$, David Galkowski ${ }^{2}$, Yuan R. Huang ${ }^{3}$, Abhijeet Joshi ${ }^{3}$, Christine Lee ${ }^{4}$, Kevin K. Leung ${ }^{5}$, \\ Gitendra Malla ${ }^{6}$, Jennifer Treanor ${ }^{7}$, Vlad Voroninski ${ }^{8}$, Andrea L. Bertozzi ${ }^{1}$, and Yen-Hsi R. Tsai ${ }^{9}$
}

\begin{abstract}
Autonomous robotic systems (observers) equipped with range sensors must be able to discover their surroundings, in an initially unknown environment, for navigational purposes. We present an implementation of a recent environmentmapping algorithm [1] based on Essentially Non-oscillatory (ENO) interpolation [2]. An economical cooperative control tank-based platform [3] is used to validate our algorithm. Each vehicle on the test-bed is equipped with a flexible caterpillar drive, range sensor, limited onboard computing, and wireless communication.
\end{abstract}

\section{INTRODUCTION}

In this paper we present an implementation of a pathplanning algorithm which allows a group of autonomous vehicles equipped with range-sensors (observers) to explore an unknown bounded region and construct the map of the explored environment. This algorithm was introduced in [1] and is based on determining visible portions of a bounded two-dimensional region from a given vantage point. To test the robustness of our algorithm, we consider the problem of mapping an unknown environment using multiple mobile inexpensive sensors where noise is an issue.

The outline of the paper is as follows. In section II we present some of the existing algorithms for generating visibility in an unknown environment and visibility based pathplanning. Then, in section III we describe the Visibility Interpolation algorithm first introduced in [1] and its extension for multiple observers. Section IV discusses environment navigation algorithm based on Visibility Interpolation. In section $\mathrm{V}$ we introduce the test-bed and the robot-vehicles used for navigation as well as the range sensors. Finally, section VI contains the results of implementation on the testbed.

\footnotetext{
${ }^{1}$ Dept. of Mathematics, University of California Los Angeles, Los Angeles, CA 90095 \{ylanda, bertozzi\}@math.ucla.edu

${ }^{2}$ Dept. of Physics, Harvard University, Cambridge, MA 02138 dgalkowsefas.harvard.edu

${ }^{3}$ Dept. of Electrical Engineering, University of California Los Angeles, Los Angeles, CA 90095 yuanh@seas.ucla.edu, abhijeet Qucla.edu

${ }^{4}$ Dept. of Mathematics, California Institute of Technology, Pasadena, CA 91125 crslecaltech.edu

${ }^{5}$ Dept. of Electrical and Computer Engineering, University of California Davis, Davis, CA 95616 kevinlgeicedepot.net

${ }^{6}$ Dept. of Computer Science, Dept. of Mathematics, Gettysburg College, Gettysburg, PA 17325 mallgi01@gettysburg.edu

${ }^{7}$ Dept. of Physics, Trinity College Dublin, Dublin 2, Ireland jenny.treanor@gmail.com

${ }^{8}$ Dept. of Computer Science, University of California Los Angeles, Los Angeles, CA 90095 vladvoro@ucla.edu

${ }^{9}$ Dept. of Mathematics, University of Texas at Austin, Austin, TX 78704 ytsai@math.utexas.edu
}

\section{PREVIOUS WORK}

In this section we provide a brief survey of related work in the area of visibility based navigation, sensing, and cooperative control. Computational geometry and combinatorics are currently the main tools for solving visibility-based navigation problems [4], [5], [6]. The combinatorial approach is mainly concerned with defining visibility on polygons and other special types of planar environments. Simplified planar polygonal environment is the main limitation of combinatorial approach.

In [7] a visibility function and obstacle boundaries are represented by level set functions [8]. This formulation is used in [9] to solve various optimization problems related to visibility. The method works on general types of environment, however, it requires a priori knowledge of the occluding objects to construct a level set representation. Such information may not be available in some real applications.

In [10], an algorithm extracting planar information from point clouds is introduced and used in mapping outdoor environment. In [11], depth to the occluding objects is estimated by a trinocular stereo vision system and is then combined with a predetermined "potential" function so that a robot can move to the desired location without crashing into obstacles.

Motivation for the visibility formulation and subsequent navigation algorithms in [1] comes from work of Tovar et al. [12], [13], [14], [15], and [16]. In [16], a single robot (observer) must be able to navigate through an unknown simply or multiply connected piecewise-analytic environment. The robot is equipped with a sensor that maps onto a circle relative locations of discontinuities in depth information (gaps) in the order of their appearance with respect to the robot's heading. Each gap corresponds to a connected portion of space that is not visible to the robot.

To navigate the environment, the robot approaches one of the gaps. No distance or angular information is utilized unlike in [1], where an additional map of the original domain in cartesian coordinates is used to aid the path planning.

The Gap Navigation Tree (GNT), described in [12], encodes paths from the current position of the robot to any place in the environment and is updated dynamically as the robot moves. The exploration is complete when all the gaps have been approached. A minimal representation of the environment is constructed in form of the dynamic tree based on gaps. In contrast, in [1], an implicit representation of the obstacles in the environment is reconstructed at the termination of the path. 
In [13] the GNT based algorithm [16] was tested on a Pioneer 2-DX platform equipped with two SICK laser range sensors which provide an omnidirectional view. The gap sensor implementation combined the data of these two sensors. Simple test environments were chosen to be within the sensor range. Wall-following capabilities were implemented to avoid collisions.

Another wall-following control algorithm is discussed in [17]. In this work, curvature-based control algorithms from [18] are tested using real range sensors. Curvature is computed from the range data obtained by SICK LMS-200 laser range sensors. Unlike range sensors used in our experiment, the range-finder in [17] has a range of $10 \mathrm{~m}$ and relative error less then $0.8 \%$.

Even with such a high precision, curvature estimates have significant inaccuracies in the absence of filtering. The noise in curvature computations is related to the computation of derivatives of the range data which are prone to noise. To deal with this problem, ENO interpolation was introduced in [1], to obtain high order representation of the range data, so that derivatives can be easily estimated away from discontinuities (see Fig. 2).

In another work [19], a multiple vehicle cooperative control algorithm is described. The model problem is extended from the classical Art Gallery Problem [6]. Here, each robot must find a location in a non-convex polygonal environment, so that each point of the environment is visible to at least one robot. In [19] the visibility-based deployment problem is solved under the assumption that all the vehicles are initially collocated.

In this paper we describe a multiple vehicle environment exploration algorithm based on a Visibility Interpolation formulation introduced in [1]. This algorithm works on general types of environment and is easy to scale for an arbitrary number of observers. As a result of the exploration a map of the environment is produced, where obstacle boundaries are represented by high order polynomial curves.

\section{VISIBILITY INTERPOLATION}

The visibility formulation from [1] is described below. It is then applied to the problem of environment exploration by single and multiple observers.

The range-sensor attached to an autonomous vehicle is used to sample data from opaque objects in the environment. The obtained point cloud is then sampled onto a sphere centered at the observing location and interpolated to accurately represent visible boundaries of occluding objects. The following construction of visibility was introduced in [1].

Assume a point cloud $P$ is uniformly sampled from the occluding surfaces in the bounded domain $\Omega$ by the range sensing device. Given a vantage point $x_{0}$ and a point $x$ in $\Omega$, let $\nu\left(x_{0}, x\right):=\left(x-x_{0}\right) /\left|x-x_{0}\right|$ be the view direction from $x_{0}$ to $x$. For any direction defined by a unit vector $p$ construct a piecewise continuous function on a unit sphere:

$\rho_{x_{0}}(p):= \begin{cases}\min _{x \in \Omega}\left\{\left|x-x_{0}\right|: \nu\left(x_{0}, x\right)=p\right\}, & \text { if exists } \\ \infty, & \text { otherwise }\end{cases}$
Define the visibility indicator

$$
\Xi\left(x, x_{0}\right):=\rho_{x_{0}}\left(\nu\left(x, x_{0}\right)\right)-\left|x-x_{0}\right|,
$$

such that $\left\{\Xi\left(x, x_{0}\right) \geq 0\right\}$ is the set of visible regions and $\left\{\Xi\left(x, x_{0}\right)<0\right\}$ is the set of invisible regions from $x_{0}$.

Enumerate all the points $y_{i} \in P$. Define a projection operator $\pi_{x_{0}}: R^{d} \rightarrow S^{d-1}$, mapping a point onto a unit sphere centered at $x_{0}$. Then define a piecewise constant approximation to $\rho_{x_{0}}$ by

$\tilde{\rho}_{x_{0}}(z):=\min \left\{\rho_{x_{0}}(z),\left|x_{0}-y_{i}\right|\right\}$, for every $y_{i} \in \pi_{x_{0}} B\left(y_{i}, \epsilon\right)$,

where $\epsilon>0$ is chosen as in [1].

Analytically, $\rho$ is piecewise continuous with jumps corresponding to the location of horizons, i.e. points where $\nu\left(x, x_{0}\right) \cdot n(x)=0, n(x)$ is outer normal vector to the occluder's boundary. Smoothness of $\rho$ in each its continuous piece corresponds to smoothness of visible portion of the occluding surface. We use discontinuity preserving Essentially Non-oscillatory (ENO) interpolation introduced by Harten et al. [2] to construct a piecewise $p$-th order polynomial approximation $\rho_{x_{0}}^{E N O(p)}$ to $\rho_{x_{0}}$ from $\tilde{\rho}_{x_{0}}$. Our approximation $\rho_{x_{0}}^{E N O(p)}$ is then used to compute derivatives on the occluding surfaces (away from the edges) and easily extract various geometric quantities, such as curvature. Fig. 1 depicts visibility map obtained via (2) and Fig. 2 illustrates corresponding $\rho^{E N O(4)}$, its derivatives, and curvature.

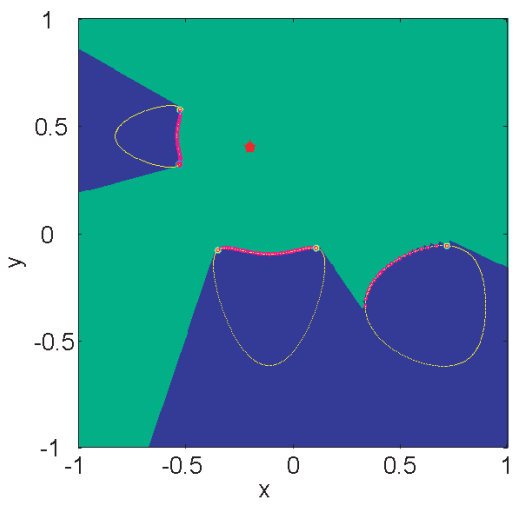

Fig. 1. Visibility map generated from artificial data: dark regions - invisible, light regions - visible, red star - vantage point $(-0.2,0.4)$, magenta circles - visible boundary, yellow circles - horizon points.

\section{APPLICATION OF VisibiLity INTERPOLATION TO NAVigation PRoblem}

In this paper we consider application of Visibility Interpolation to the problem of exploration of an unknown bounded two-dimensional region which may contain obstacles. Similarly to [16], we navigate in the environment by approaching one of the edges corresponding to horizons of the visibility function $\rho$ defined on a unit circle. The shape of obstacles may be arbitrary. During exploration we construct a map of "seen" environment, i.e. boundaries of obstacles.

We set the following restrictions on the path traveled by the observer: the path should be continuous and consist of 

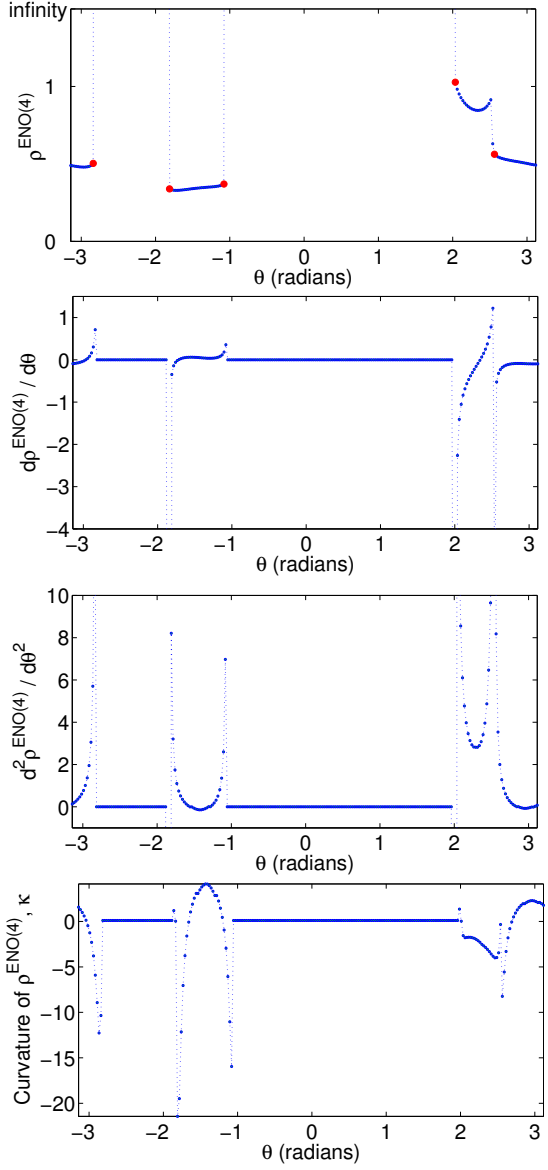

Fig. 2. ENO interpolated visibility function $\rho^{\mathrm{ENO}(4)}(\theta)$ corresponding to Fig. 1 with edges marked by red circles; first and second derivatives of $\rho^{\mathrm{ENO}(4)}(\theta)$, and the curvature.

discrete steps; the number of steps should be finite, and the total distance traveled must be finite. Below we first introduce the basic algorithm for a single observer. Then we describe its extension to multiple observers.

\section{Algorithm 1 (Single observer).}

1) For the given $x_{0}$ outside the occluding objects construct the visibility function $\rho_{x_{0}}(\theta)$;

2) Find all the discontinuities (edges) on the $\left(\theta, \rho_{x_{0}}(\theta)\right)$ map and choose the edge to approach, say, in the direction of $\theta_{e}$ (store unexplored edges in a list). The choice of an edge depends on particular aspects of the problem and will be discussed below.

If $\rho_{x_{0}}\left(\theta_{e}\right)<\rho_{x_{0}}\left(\theta_{e}+\delta\right)$, choose the direction $\theta_{e}+\delta$

Else choose the direction $\theta_{e}-\delta$;

here $\delta$ is chosen so that the observer does not approach the obstacle closer then some fixed distance parameter $\lambda$.

3) Move $x_{0}$ along the chosen direction by amount $r=$ $\min \left\{\tan \left(\frac{\pi}{3}\right) \frac{1}{\kappa}, d\right\}$, where $\kappa$ is the curvature of an edge and $d$ is a parameter controlling the maximum stepsize. If $\kappa=0$, shift $x_{0}$ by small amount to see the next edge.
4) Finish when all the edges are "removed" from the list; otherwise proceed to Step 1 with current location of $x_{0}$.

The above algorithm always converges. Its optimality depends on the choice of edge in Step 2. In [1], the nearest edge to the observer at $x_{0}$ is chosen, as opposed to the random edge in [16]. Another alternative would be to approach the edge with corresponding largest curvature $\kappa$, which maximizes the area revealed.

In our experiments, the choice of the next edge to approach in Step 2 is dictated by the specifics of the sensor design described in section $\mathrm{V}$. We prefer to move around the obstacles in the counter-clockwise fashion to minimize the effects of errors produced by the sensors. Thus, in Step 2 of Algorithm 1 we choose the right-most edge of the object.

Consider the following extension for multiple observers. Let $\left\{x_{j}\right\}_{j=1}^{n}$ be a set of observing locations. Also, let $\Xi_{j}$ be a visibility indicator map defined by (2) corresponding to $x_{j}$. In addition, let $\Theta_{j}=\left\{\theta_{j, 1}, \ldots, \theta_{j, k}\right\}$ be a set of $e$ dges visible from the vantage point at $x_{j}$. The algorithm for multiple observers is as follows.

\section{Algorithm 2 (Multiple observers).}

1) For each $x_{j}$ outside the occluding objects construct the visibility function $\rho_{x_{j}}(\theta)$

2) Compute $\Xi=\max _{j}\left\{\Xi_{j}\right\}$;

3) Find the set of edges $\Theta_{j}$ corresponding to each $x_{j}$. For each $j$, exclude those $\theta_{j, k}$ for which $\Xi \geq 0$;

4) If there remain edges for observer at $x_{j}$ to approach, do so as in Algorithm 1, Steps 2 and 3;

Else move observer at $x_{j}$ in the direction perpendicular to the direction of the nearest $x_{i}$ to see new edges;

5) Finish when all the edges are "explored"; otherwise go to Step 1 with current locations $x_{j}$.

Note that in Step 3 of the above algorithm we are excluding those edges corresponding to $x_{j}$, which are visible by another observer $x_{i}$ and thus do not need to be further explored. The perpendicular move in Step 4 is chosen to maximize chance of "seeing" more new area.

We would like to remark on different modes of execution of Algorithm 2. In concurrent mode all observers process sensor data simultaneously. This way, the next vantage point of each observer depends only on their previous positions. In sequential mode the observers are ordered as a sequence, and only one may move at a time. In this situation, position of the next observer depends on new positions of previous observers. The ordering may change according to decision to optimize joint visibility. Further details on these algorithms will be reported in a forthcoming paper [20]. In our experiments, we implement the concurrent mode.

Results of implementation of Algorithm 2 will be discussed in detail in section VI.

\section{Test-Bed And Range SEnsors}

The results in this paper were obtained using the second generation [3] of an economical micro car test-bed developed 
in [21]. The purpose of the test-bed is to design a cost effective platform to study cooperative control strategies. The dimensions of the test-bed floor are $200 \times 160 \mathrm{~cm}$. The second generation vehicles communicate at $30 \mathrm{~Hz}$ and possess onboard processing and onboard range sensing. Tank-based vehicles with caterpillar-style drive are used to allow for a zero turning radius. The tank has dimensions $7 \times 3.8 \times 4.6$ $\mathrm{cm}$ and weighs $65 \mathrm{~g}$ with batteries. Such a tank is depicted in Fig. 3. The position of the vehicles is tracked by overhead cameras. An off-board computer is used for communication with the overhead cameras and for processing sensor data from the vehicles. All the basic motion maneuver, sensor acquisition, and communication routine is processed onboard by a $16 \mathrm{MHz}$ Atmel (Atmega 8) microprocessor. The tank drives two belts independently, resulting in turns of arbitrary radius, while moving forward and backward. One can obtain more details about the test-bed and the vehicles in [3].

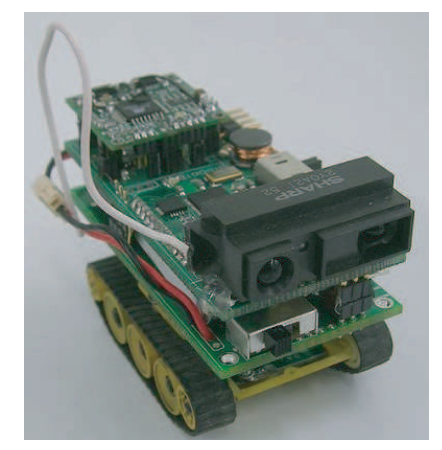

Fig. 3. Tank with the attached sensor.

Now we shall describe the range sensors used in our experiments. We work with sensors manufactured by Sharp (model 2YOAO2 F58) of range $20-150 \mathrm{~cm}$. The sensors are equipped with a PSD onto which the light is focused. IR EM radiation is emitted via LED at the front of the sensor. The wavelength range in use is $850 \mathrm{~nm} \pm 70 \mathrm{~nm}$. The halfintensity angle of the device is $1.5^{\circ}$. See Fig. 4 for schematic sensor layout.

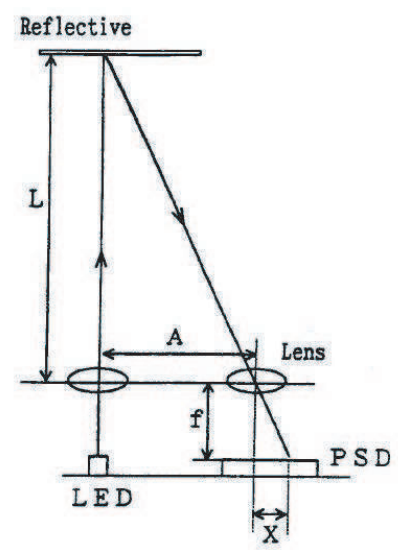

Fig. 4. Schematic sensor layout and ray patterns.
The sensor must be mounted so that the line between LED output and receiver is parallel to the ground (see Fig. 3) to minimize the effect of sensor sensitivities to the boundaries, i.e. sharp differences in texture or color of the object. For simplicity, we have idealized our environment by covering the occluding objects with white paper for uniformity in color, texture, and reflected ambient light.

Here we describe the process of sensor data calibration. The sensor takes readings at a rate $25 \mathrm{~Hz}$. Sensor readings are produced by Analog Digital Converter (ADC), which outputs values proportional to voltage output $(\mathrm{V} \times 204.8)$. The raw data obtained from the sensor over a period of several seconds is depicted in Fig. 5. We use the most frequent reading as the value at current position. In Fig. 6 we plot values at given distances from the object measured along the normal to the surface of an object.

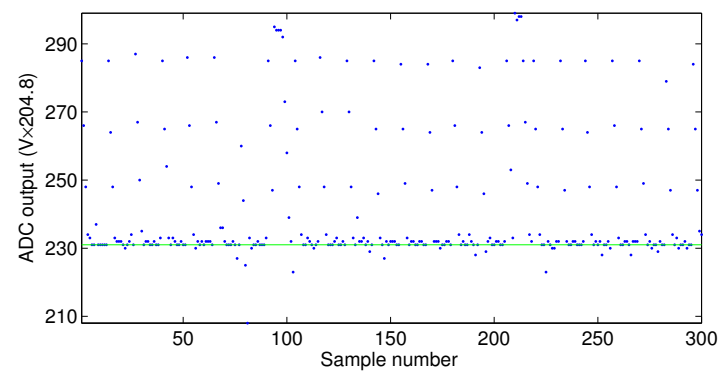

Fig. 5. Sensor ADC output $60 \mathrm{~cm}$ away from the object; green line corresponds to the most frequent value.

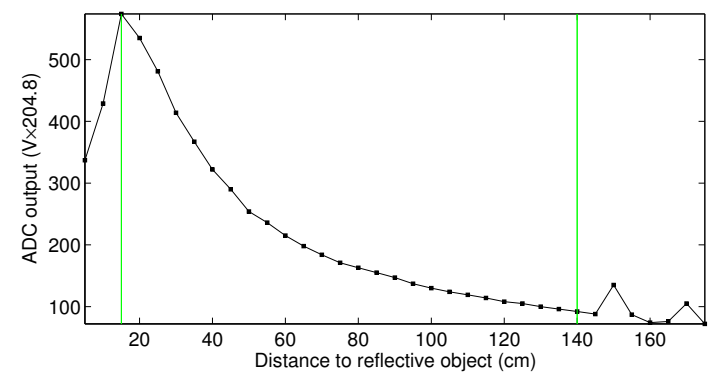

Fig. 6. Sensor ADC output corresponding to distance to reflective object measured along the normal to the surface; green vertical lines mark working sensor range.

In Fig. 7 we show several range curves constructed from different angles to the surface of the object. As one can see from Fig. 7, the range calibration curves are shifted with respect to one another for different viewing angles (upward, when the object is viewed from the right, downward, when object is viewed from the left). This results in the same sensor output value for two different sensor positions. For example, sensor output at a distance of $90 \mathrm{~cm}$ from the object at an angle $-85^{\circ}$ to the normal to the surface is the same as the sensor output at a distance of $45 \mathrm{~cm}$ at an angle $+75^{\circ}$ and yet the same as the output at a distance of $60 \mathrm{~cm}$ along the normal to the surface.

If we take as a reference the range curve measured along the normal to the surface of reflective object, we obtain 


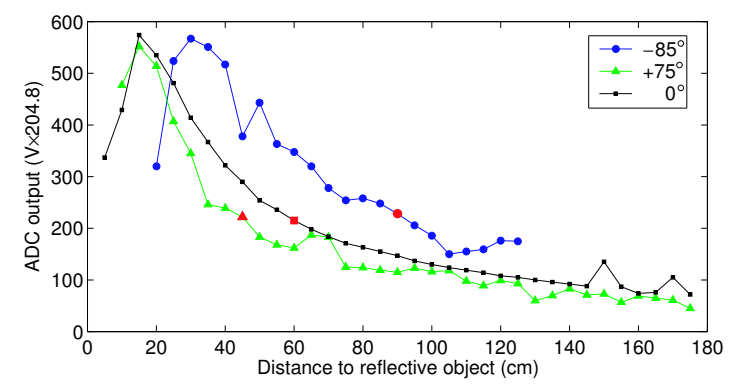

Fig. 7. Sensor ADC output corresponding to distance to reflective object measured along different angles to the normal to the surface; red marks correspond to points on the range curves with similar sensor output.

inaccuracies when looking at an object from a different angle. For example, one can see from Fig. 8 the tilt in the measured surface position with respect to the actual one.

The results may be improved by taking several measurements along a given direction. This way we can find a matching range curve from which we can deduce the distance to the object and the incident angle. However, this solution is too expensive and thus we did not implement it.

In addition, we note that the shift is only significant when looking at an object from the right. Thus, a path-planning algorithm is modified with a bias towards moving in a counter-clockwise manner. See Fig. 8 for an example.

\section{Results And CONCLUSION}

In summary, we implement a multi-vehicle environment mapping algorithm based on a Visibility Interpolation formulation introduced in [1]. The algorithm does not require any shape priors for the occluding objects. We use two boxes as our sample obstacles for easy representation. The positions, shapes, and quantities of obstacles are unknowns.

Two tank-based vehicles equipped with the range sensors are initially positioned on the test-bed floor outside the obstacles. Each tank makes a $360^{\circ}$ sweep to gather range data from its surrounding environment. About 80 samples are taken in one sweep. Each sweep takes less then a minute to complete. Then, a visibility map and next position of each vehicle is computed off-board based on sensor output. The next observer's position is transmitted to the robots and they proceed to collect data from new vantage point. This process is repeated until the whole region has been explored as in Algorithm 2 above. In the example, exploration took two steps by each observer.

The obtained range data is fit to the range calibration curve in Fig. 6 via cubic interpolation. Then the data is processed in the following way. Whenever we get a hit which is outside of the range of the sensor or its $x, y$ position is outside the test-bed floor, we assign the value of "infinity", which is set to be at $120 \mathrm{~cm}$.

Joint visibility maps after each step are depicted in Fig. 8. Actual obstacle boundaries are represented by yellow lines on each figure. Red stars represent positions of the robots after each step. The red lines mark the path of each vehicle up until its current location. Dark regions are invisible at current step and lighter regions are visible. Magenta circles represent shadow boundary obtained via ENO high order interpolation of the obtained range data. Black circles represent horizon points which will be approached in the next step.

The complete visibility map is depicted in Fig. 9. It is constructed by taking the union of visibility maps of all observers at all steps. From this map, one can estimate the quantity, size, and locations of the obstacles. However, the boundaries are not accurately represented due to low sensor accuracy and small number of samples.

As was mentioned above, the results may be improved by correcting for the angle of incidence of the IR beam. Overall, the quality of the results is satisfactory taking into account hardware limitations.
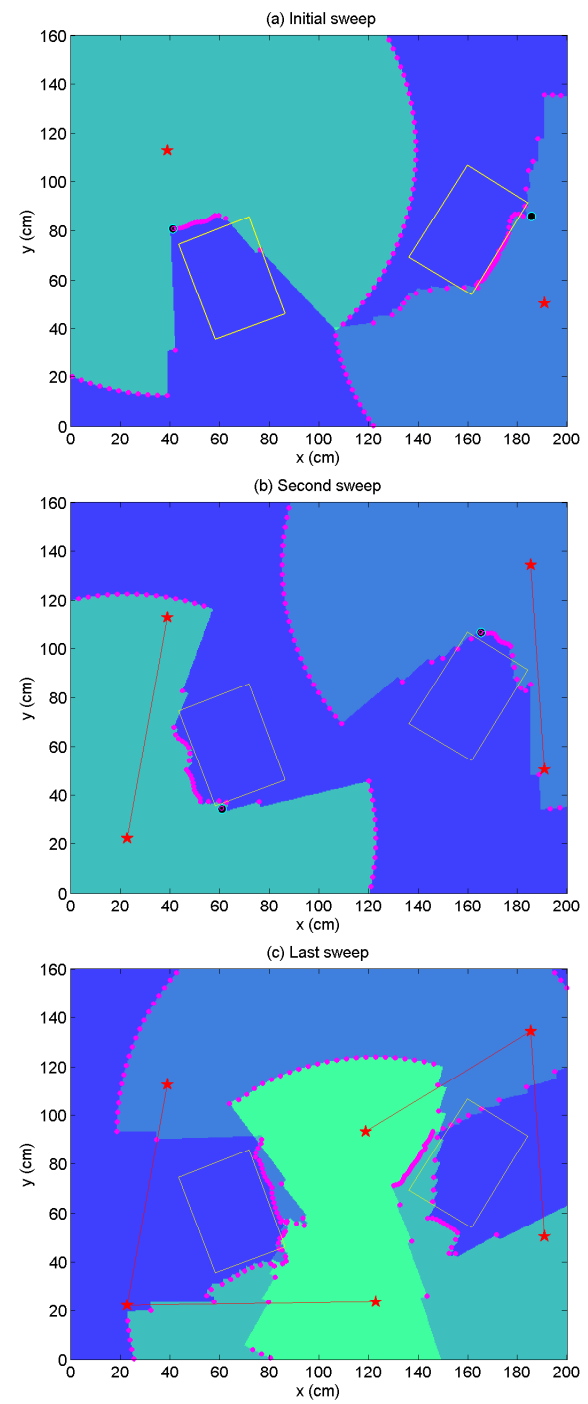

Fig. 8. Exploration of environment with 2 observers. Red stars are observers' positions; magenta circles are the sensor output converted to range data; big dark circles are the next edges to be approached; yellow boxes are the actual obstacle outlines; dark regions are currently invisible; light regions are currently visible. 


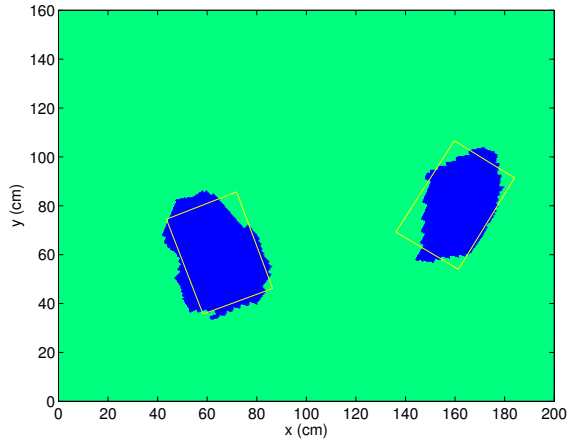

Fig. 9. Map resulting from the environment exploration. Dark regions are invisible and light regions are visible. Yellow boxes are the actual outlines of obstacles.

\section{ACKNOWLEDGEMENTS}

This research was supported as part of the Research in Industrial Projects for Students (RIPS) program at the Institute for Pure and Applied Mathematics (IPAM), and funded by NSF grant DMS-0439872 and NSA grant H9823006-1-0057. We would like to thank Matthew Sottile who was a RIPS industrial mentor from Los Alamos National Laboratory. Additionally, this research was supported by NSF grant DMS-0601395, ARO MURI grant 50363-MAMUR, ONR grant N000140610059, ARO grant W911NF05-1-0112, and NSF grant DMS-0513394.

\section{REFERENCES}

[1] Y. Landa, R. Tsai, and L.-T. Cheng, "Visibility of point clouds and mapping of unknown environments", Advanced Concepts for Intelligent Vision Systems, ACIVS 2006, Sept 18-21, 2006, University of Antwerp, Belgium (preprint available as UCLA CAM report 06-16).

[2] A. Harten, B. Engquist, S. Osher, S.R. Chakravarthy, "Uniformly high order accurate essentially nonoscillatory schemes, III," Journal of Computational Physics, 71, (1987), 231-303.

[3] K. K. Leung, C. H. Hsieh, Y. R. Huang, A. Joshi, V. Voroninski, and A. L. Bertozzi, "A second generation microvehicle testbed for cooperative control and sensing strategies", submitted to ACC (2007).

[4] W.-P. Chin and S. Ntafos, "Shortest watchman routes in simple polygons", Discrete Comput. Geom., 6 (1991), 9-31.
[5] J. E. Goodman, J. O'Rourke, editors "Handbook of discrete and computational geometry", CRC Press LLC, Boca Raton, FL; Second Edition, April (2004).

[6] J. Urrutia. "Art gallery and illumination problems", in J. R. Sack and J. Urrutia, editors, "Handbook of Computational Geometry", 973-1027, (2000).

[7] Y.-H. R. Tsai, L.-T. Cheng, S. Osher, P. Burchard, G. Sapiro, "Visibility and its dynamics in a PDE based implicit framework" Journal of Computational Physics, 199, 260-290, (2004).

[8] S. Osher, J. A. Sethian, "Fronts propagating with curvature-dependent speed: algorithms based on HamiltonJacobi formulations", Journal of Computational Physics 79 (1) 12-49, (1988).

[9] L.-T. Cheng and R. Tsai, "Visibility optimizations using variational approaches", Communications of Mathematical Sciences, 3, (3) (2005).

[10] F. Wolf, A. Howard, and G. S. Sukhatme. "Towards geometric 3D mapping of outdoor environments using mobile robots", IEEE/RSJ International Conference on Intelligent Robots and Systems (IROS),1258-1263, (2005).

[11] D. Murray and C. Jennings. "Stereo vision based mapping for a mobile robot", Proc. IEEE Conf. on Robotics and Automation, (1997).

[12] B. Tovar, L. Guilamo, and S.M. LaValle, "Gap navigation trees: minimal representation for visibility-based tasks", Proc. Workshop on the Algorithmic Foundations of Robotics, (2004).

[13] B. Tovar, S. M. LaValle, and R. Murrieta, "Optimal navigation and object finding without geometric maps or localization", Proc. IEEE International Conference on Intelligent Robots and Systems, (2003).

[14] B. Tovar, S. M. LaValle, and R. Murrieta, "Locally-optimal navigation in multiply-connected environments without geometric maps", IEEE/RSJ International Conference on Intelligent Robots and Systems, (2003).

[15] B. Tovar, S. M. LaValle, and R. Murrieta, "Optimal navigation and object finding without geometric maps or localization", IEEE International Conference on Robotics and Automation, (2003).

[16] B. Tovar, R. Murrieta-Cid, and S. M. LaValle "Distance-optimal navigation in an unknown environment without sensing distances", IEEE Transactions on Robotics, (2006). Under review.

[17] F. Zhang, A. O'Connor, D. Luebke, and P. S. Krishnaprasad, "Experimental study of curvature-based control laws for obstacle avoidance", Proceedings of the 2004 IEEE International Conference on Robotics and Automation, New Orleans, LA, April (2004).

[18] F. Zhang, E. Justh, and P. S. Krishnaprasad, "Steering control, curvature and Lyapunov navigation", preprint, (2003).

[19] A. Ganguli, J. Cortes, F. Bullo, "Distributed deployment of asynchronous guards in art galleries", Proceedings of the 2006 American Control Conference, Minneapolis, MN, June 14-16, (2006).

[20] Y. Landa, R. Tsai, "Mapping unknown environments from range data using multiple observers", (2006).

[21] C. H. Hsieh, Y.-L. Chuang, Y. Huang, K. K. Leung, A. L. Bertozzi, and E. Frazzoli, "An economical micro-car testbed for validation of cooperative control strategies", Proceedings of the 2006 American Control Conference, Minneapolis, MN, June 14-16, 1446-1451, (2006). 